\title{
ON A THEOREM OF SERRET ON CONTINUED FRACTIONS
}

\author{
PALOMA BENGOECHEA
}

\begin{abstract}
A classical theorem in continued fractions due to Serret shows that for any two irrational numbers $x$ and $y$ related by a transformation $\gamma$ in $\operatorname{PGL}(2, \mathbb{Z})$ there exist $s$ and $t$ for which the complete quotients $x_{s}$ and $y_{t}$ coincide. In this paper we give an upper bound in terms of $\gamma$ for the smallest indices $s$ and $t$.
\end{abstract}

A classical theorem of Serret Ser66] states that the standard continued fraction expansions of two irrational numbers $x=\left[n_{0}, n_{1}, \ldots\right]$ and $y=\left[m_{0}, m_{1}, \ldots\right]$ are the same after a finite number of steps if and only if $x$ and $y$ are $\operatorname{PGL}(2, \mathbb{Z})$-equivalent. Serret's theorem does not quantify how large the smallest indices $s$ and $t$ have to be such that the complete quotients $x_{s}=\left[n_{s}, n_{s+1}, \ldots\right]$ and $y_{t}=\left[m_{t}, m_{t+1}, \ldots\right]$ coincide. The more complicated the matrix $\gamma$ relating $x$ and $y$ is, the larger $s$ or $t$ becomes. A quantification of this fact, namely bounding $s$ and $t$ in terms of $\gamma$, is the goal of this paper. The main theorem, in Section 2, gives an upper bound for the smallest $s$ and $t$ such that $x_{s}=y_{t}$. The bound we give is the best possible in the sense that for each $\gamma \in \operatorname{PGL}(2, \mathbb{Z})$ one can construct many real numbers $x$ for which $s$ or $t$ almost attain the bound, and for many matrices $\gamma$ the bound is actually attained. In order to prove the theorem, in the first section we introduce the set $\Gamma(x)$ of transformations of $\operatorname{PGL}(2, \mathbb{Z})$ related to the continued fraction expansion of the real number $x$. We characterize it by certain linear inequalities in Proposition 1.2. Sets of transformations related to other continued fraction algorithms have been characterized in [KU10] in a similar way.

\section{Linear ineQUALities CHARACTERIZING THE CONTINUED FRACTION TRANSFORMATIONS}

We denote by $\Gamma$ the group $\operatorname{PGL}(2, \mathbb{Z})$ and by $\varepsilon$ and $T$ the transformations

$$
\varepsilon=\left(\begin{array}{ll}
0 & 1 \\
1 & 0
\end{array}\right), \quad T=\left(\begin{array}{ll}
1 & 1 \\
0 & 1
\end{array}\right)
$$

Key words and phrases. Continued fractions, $\mathrm{PGL}(2, \mathbb{Z})$-equivalent numbers. Mathematics Subject Classification: 11A55. 
that correspond to the inversion and the translation for the usual action on the projective line

$$
\left(\begin{array}{ll}
a & b \\
c & d
\end{array}\right) x:=\frac{a x+b}{c x+d}
$$

The positive continued fraction of a real number $x$,

$$
x=n_{0}+\frac{1}{n_{1}+\frac{1}{n_{2}+\frac{1}{\ddots}}} \quad\left(n_{0} \in \mathbb{Z}, n_{i} \geq 1 \forall i \geq 1\right),
$$

also denoted by $x=\left[n_{0}, n_{1}, \ldots\right]$, is given by the algorithm

$$
\begin{aligned}
& x_{0}=x, \\
& n_{i}=\left\lfloor x_{i}\right\rfloor, \quad x_{i+1}=\frac{1}{x_{i}-n_{i}}=\varepsilon T^{-n_{i}}\left(x_{i}\right) \quad \text { if } x_{i} \notin \mathbb{Z} \quad(i \geq 0) .
\end{aligned}
$$

If $x_{i} \in \mathbb{Z}$, the algorithm stops after $n_{i}=\left\lfloor x_{i}\right\rfloor$. Clearly each complete quotient $x_{i}=\left[n_{i}, n_{i+1}, \ldots\right]$ is the image of $x$ by a matrix $\gamma_{i}=\gamma_{i, x} \in \Gamma$, given explicitly by

$$
\gamma_{0}:=\mathrm{Id}, \quad \gamma_{i}:=\left(\begin{array}{cc}
0 & 1 \\
1 & -n_{i-1}
\end{array}\right) \cdots\left(\begin{array}{cc}
0 & 1 \\
1 & -n_{0}
\end{array}\right) \quad(i \geq 1)
$$

and recursively by

$$
\gamma_{0}=\mathrm{Id}, \quad \gamma_{i+1}=\varepsilon T^{-n_{i}} \gamma_{i} \quad(i \geq 0) .
$$

A key idea is to replace the sequence $\left(\gamma_{1}, \gamma_{2}, \gamma_{3}, \ldots\right)$ of elements in $\Gamma$ by the unordered set

$$
\Gamma(x)=\left\{\gamma_{1}, \gamma_{2}, \gamma_{3}, \ldots\right\} \subset \Gamma .
$$

When $x \in \mathbb{R} \backslash \mathbb{Q}$, the set $\Gamma(x)$ is infinite, whereas it is finite if $x \in \mathbb{Q}$. If $x=\left[n_{0}, \ldots, n_{t}\right]$, then

$$
\Gamma(x)=\left\{\gamma_{1}, \ldots, \gamma_{t+1}\right\} .
$$

The $i$-th convergent of $x$ is denoted by $\frac{p_{i}}{q_{i}}=\left[n_{0}, \ldots, n_{i}\right]$. The integers $p_{i}$ and $q_{i}$ satisfy the recurrence

$$
\begin{aligned}
& p_{-2}=0, \quad p_{-1}=1, \quad p_{i}=n_{i} p_{i-1}+p_{i-2} \quad(i \geq 0), \\
& q_{-2}=1, \quad q_{-1}=0, \quad q_{i}=n_{i} q_{i-1}+q_{i-2} \quad(i \geq 0),
\end{aligned}
$$

the equation

$$
p_{i+1} q_{i}-p_{i} q_{i+1}=(-1)^{i}
$$

and the inequalities

$$
q_{i} \geq q_{i-1} \geq 0 \quad(i \geq 0), \quad q_{i}>q_{i-1}>0 \quad(i \geq 2)
$$




$$
\left|p_{i}\right| \geq\left|p_{i-1}\right| \quad(i \geq 2), \quad\left|p_{i}\right|>\left|p_{i-1}\right| \quad(i \geq 4) .
$$

It is well known that any rational number $p / q$ satisfying

$$
\left|\frac{p}{q}-x\right|<\frac{1}{2 q^{2}}
$$

is a convergent of $x$.

The numbers $\delta_{i}(i \geq-1)$ defined by

$$
\delta_{i}=(-1)^{i}\left(p_{i-1}-q_{i-1} x\right)
$$

satisfy the recurrence

$$
\delta_{-1}=x, \quad \delta_{0}=1, \quad \delta_{i+1}=-n_{i} \delta_{i}+\delta_{i-1},
$$

and the inequalities $1=\delta_{0}>\delta_{1}>\ldots \geq 0$. If $x$ is rational, then $x_{i}=p_{i} / q_{i}$ for some $i$ and the recurrence stops with $\delta_{i+1}=0$; if $x$ is irrational, the $\delta_{i}$ are all positive and converge to 0 with exponential rapidity. One has

$$
n_{i}=\left\lfloor\frac{\delta_{i-1}}{\delta_{i}}\right\rfloor \quad \text { if } \delta_{i} \neq 0
$$

and

$$
\gamma_{i}^{-1}=\left(\begin{array}{cc}
p_{i-1} & p_{i-2} \\
q_{i-1} & q_{i-2}
\end{array}\right), \quad \gamma_{i}\left(\begin{array}{c}
x \\
1
\end{array}\right)=\left(\begin{array}{c}
\delta_{i-1} \\
\delta_{i}
\end{array}\right) \text {. }
$$

Lemma 1.1. If $r / t$ and $s / u$ are two rational numbers such that $t, u>0$, $\frac{r}{t} \leq x \leq \frac{s}{u}$ and $r u-s t= \pm 1$, then $r / t$ or $s / u$ is a convergent of $x$.

Proof. Let $x$ be an element in $\left[\frac{r}{t}, \frac{s}{u}\right]$. If one of the inequalities

$$
\left|\frac{r}{t}-x\right|<\frac{1}{2 t^{2}} \quad \text { or } \quad\left|\frac{s}{u}-x\right|<\frac{1}{2 u^{2}}
$$

is satisfied, then $r / t$ or $s / u$ is a convergent of $x$. If not, then

$$
\frac{1}{t u}=\left|\frac{r}{t}-\frac{s}{u}\right|=\left|\frac{r}{t}-x\right|+\left|\frac{s}{u}-x\right| \geq \frac{1}{2 t^{2}}+\frac{1}{2 u^{2}} .
$$

The inequality above can only hold if $t=u=1$, in which case $s=r+1$, so $\left[\frac{r}{t}, \frac{s}{u}\right]=[r, r+1]$, and then either $\frac{s}{u}=x=\frac{p_{0}}{q_{0}}$ or $\frac{r}{t}=\lfloor x\rfloor=\frac{p_{0}}{q_{0}}$.

Throughout, for any pair of real numbers $a, b$, we denote by $|a, b|$ the interval $[a, b]$ if $a \leq b$ or $[b, a]$ if $b<a$.

Proposition 1.2. For any real number $x$, the set $\Gamma(x)$ equals $W-$ $\left(W_{1} \cup W_{2}\right)$, where

$$
W=\{\gamma \in \Gamma \mid-1 \leq \gamma(\infty) \leq 0, \gamma(x)>1\}
$$


and

$$
\begin{gathered}
W_{1}=\{\gamma \in W \mid \gamma(\infty)=0, \operatorname{det}(\gamma)=1\}, \\
W_{2}=\{\gamma \in W \mid \gamma(\infty)=-1, \operatorname{det}(\gamma)=-1\} .
\end{gathered}
$$

The sets $W_{1}$ and $W_{2}$ have respectively exactly one and at most one element. (The inequality $\gamma(x)>1$ in the definition of $W$ includes $\gamma(x)=\infty$.)

Proof. One easily checks that

$$
\begin{aligned}
& W_{1}=\left\{\left(\begin{array}{cc}
0 & -1 \\
1 & -1-n_{0}
\end{array}\right)\right\},
\end{aligned}
$$

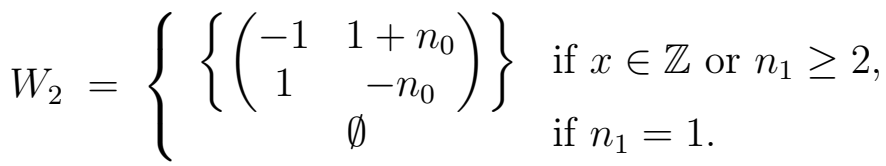

This proves the second statement. It is also easy to see that $\Gamma(x) \subseteq$ $W-\left(W_{1} \cup W_{2}\right)$. Indeed, $\gamma_{i}(x)=\frac{\delta_{i-1}}{\delta_{i}}>1$ and $\gamma_{i}(\infty)=-\frac{q_{i-2}}{q_{i-1}}$ for $i \geq 1$ together with the inequalities (2) imply $\gamma_{i} \in W-\left(W_{1} \cup W_{2}\right)$.

Therefore we only have to show that $W \subseteq \Gamma(x) \cup W_{1} \cup W_{2}$. Let $\gamma \in \Gamma$ satisfy

$$
-1 \leq \gamma(\infty)<0, \quad \gamma(x)>1
$$

Let

$$
\gamma^{-1}=\left(\begin{array}{cc}
r & s \\
t & u
\end{array}\right), \quad \gamma=\left(\begin{array}{cc}
u & -s \\
-t & r
\end{array}\right) .
$$

The conditions $\gamma(\infty)<0$ and $\gamma(x)>0$ imply that $u$ and $t$ have the same sign, as well as $u x-s$ and $-t x+r$, so $x \in\left|\frac{r}{t}, \frac{s}{u}\right|$. By Lemma 1.1. $r / t$ or $s / u$ is a convergent of $x$.

If $r / t$ is a convergent of $x$, then $\gamma$ and $\gamma^{-1}$ are of the form

$$
\gamma^{-1}=\left(\begin{array}{cc}
p_{i} & p_{i-1}+k p_{i} \\
q_{i} & q_{i-1}+k q_{i}
\end{array}\right), \quad \gamma=\left(\begin{array}{cc}
q_{i-1}+k q_{i} & -p_{i-1}-k p_{i} \\
-q_{i} & p_{i}
\end{array}\right)
$$

$\mathrm{Or}$

$$
\gamma^{-1}=\left(\begin{array}{cc}
p_{i} & -p_{i-1}-k p_{i} \\
q_{i} & -q_{i-1}-k q_{i}
\end{array}\right), \quad \gamma=\left(\begin{array}{cc}
-q_{i-1}-k q_{i} & p_{i-1}+k p_{i} \\
-q_{i} & p_{i}
\end{array}\right)
$$

with $k \in \mathbb{Z}$ and $i \geq 0$. In the case (4), since $q_{i-1} \leq q_{i}$, we have that $-1 \leq \gamma(\infty)=-\frac{q_{i-1}}{q_{i}}-k<0$ if and only if $k=0$ and $i \geq 1$, or $k=1$ and $i=0$. Moreover, if $x \notin \mathbb{Z}$, then $\gamma(x)=\frac{\delta_{i}}{\delta_{i+1}}-k>1$ if and only if $k \leq n_{i+1}-1$ because $\left\lfloor\frac{\delta_{i}}{\delta_{i+1}}\right\rfloor=n_{i+1}$. Hence $-1 \leq \gamma(\infty)<0$ and 
$\gamma(x)>1$ if and only if $k=0$ and $i \geq 1$ or $x \in \mathbb{Z}, k=1$ and $i=0$, or $n_{1} \geq 2, k=1$ and $i=0$. In the first case

$$
\gamma=\left(\begin{array}{cc}
q_{i-1} & -p_{i-1} \\
-q_{i} & p_{i}
\end{array}\right)=\gamma_{i+1} \quad(i \geq 1)
$$

in the other two cases

$$
\gamma=\left(\begin{array}{cc}
-1 & 1+n_{0} \\
1 & -n_{0}
\end{array}\right) \in W_{2}
$$

In the case (5), if $-1 \leq \gamma(\infty)=\frac{q_{i-1}}{q_{i}}+k<0$, then $k<0$ and $\gamma(x)=-\frac{\delta_{i}}{\delta_{i+1}}+k<0$, so $\gamma \notin W$.

If $s / u$ is a convergent of $x$, then $\gamma$ and $\gamma^{-1}$ are of the form

$$
\gamma^{-1}=\left(\begin{array}{cc}
p_{i-1}+k p_{i} & p_{i} \\
q_{i-1}+k q_{i} & q_{i}
\end{array}\right), \quad \gamma=\left(\begin{array}{cc}
q_{i} & -p_{i} \\
-q_{i-1}-k q_{i} & p_{i-1}+k p_{i}
\end{array}\right)
$$

or

(7) $\quad \gamma^{-1}=\left(\begin{array}{cc}-p_{i-1}-k p_{i} & p_{i} \\ -q_{i-1}-k q_{i} & q_{i}\end{array}\right), \quad \gamma=\left(\begin{array}{cc}q_{i} & -p_{i} \\ q_{i-1}+k q_{i} & -p_{i-1}-k p_{i}\end{array}\right)$

with $k \in \mathbb{Z}$ and $i \geq 0$. In the case (6),$\gamma(x)=\frac{\delta_{i+1}}{\delta_{i}-k \delta_{i+1}}>1$ if and only if $k=n_{i+1}$. If $k=n_{i+1}$, then $-1 \leq \gamma(\infty)=-\frac{q_{i}}{q_{i+1}}<0$. Hence

$$
\gamma=\left(\begin{array}{cc}
q_{i} & -p_{i} \\
-q_{i+1} & p_{i+1}
\end{array}\right)=\gamma_{i+2} \quad(i \geq 0)
$$

In the case (17), if $-1 \leq \gamma(\infty)=\frac{q_{i}}{q_{i-1}+k q_{i}}<0$, then $k<0$ and $\gamma(x)=\frac{\delta_{i+1}}{-\delta_{i}+k \delta_{i+1}} \leq 0$, so $\gamma \notin W$.

Finally, let $\gamma=\left(\begin{array}{ll}0 & 1 \\ 1 & u\end{array}\right)$ be the unique element of $W$ satisfying $\gamma(\infty)=0$ and $\operatorname{det}(\gamma)=-1$. We have $1<\gamma(x)=\frac{1}{x+u}$ if and only if $0<x+u<1$, namely $u=-\lfloor x\rfloor$, and $\gamma=\gamma_{1} \in \Gamma(x)$.

\section{MAIN THEOREM}

We recall the classic theorem of Serret about continued fraction expansions of equivalent numbers (see [Ser66]): 
Theorem 2.1 (Serret). Two irrational numbers $x$ and $y$ are $\Gamma$-equivalent if and only if there exist $s, t \geq 0$ such that $x_{s}=y_{t}$.

Theorem 2.1 (or its proof) does not give any bound independent of $x$ and $y$ for the indexes $s$ and $t$. The result we state below gives the best possible bound for the index $s$ in terms of the matrix relating $x$ and $y$ (see Remark 2.3).

We introduce the convention that $\infty$ has zero partial quotients in the rest of the paper.

Theorem 2.2. Let $\gamma \in \Gamma$ and let $r$ be the number of partial quotients of $\gamma^{-1}(\infty)$. For every real number $x$ there exists an index $s \leq r+3$ such that the complete quotient $x_{s}$ is also a complete quotient of $\gamma(x)$.

Of course the bound for $t$ is obtained in the same way in terms of the number of partial quotients of $\gamma(\infty)$.

Proof. Let $y=\gamma(x)$. Since

$$
x_{s}=\gamma_{s, x}(x) \quad \text { and } \quad y_{t}=\gamma_{t, y}(y)=\left(\gamma_{t, y} \gamma\right)(x),
$$

we must prove that there exists $s \leq r+3$ such that $\gamma_{s, x}=\gamma_{t, y} \gamma$ for some $t \geq 1$. In other words, we prove next that, if $\gamma_{i, x} \neq \gamma_{t, y} \gamma$, then $i \leq r+2$. Let $i \geq 0$ be such that $\gamma_{i, x} \gamma^{-1} \notin \Gamma(y)$. Proposition 1.2 implies one of the following conditions:

$$
\gamma_{i, x} \gamma^{-1}(\infty) \geq 0 \quad \text { or } \quad \gamma_{i, x} \gamma^{-1}(\infty) \leq-1 .
$$

Suppose $\gamma^{-1}(\infty) \neq \infty$ and set $p / q=\gamma^{-1}(\infty)$ with $(p, q)=1$. If the first inequality in (8) is satisfied, we have

$$
\gamma_{i, x}\left(\frac{p}{q}\right)=\frac{q_{i-2} p-p_{i-2} q}{-q_{i-1} p+p_{i-1} q} \geq 0
$$

which implies $\frac{p}{q} \in\left|\frac{p_{i-2}}{q_{i-2}}, \frac{p_{i-1}}{q_{i-1}}\right|$. Then by Lemma 1.1 we have that either $p_{i-1} / q_{i-1}$ or $p_{i-2} / q_{i-2}$ is a convergent of $p / q$. Similarly, if the second inequality in (8) is satisfied, we have

$$
\gamma_{i, x}\left(\frac{p}{q}\right)=\frac{q_{i-2} p-p_{i-2} q}{-q_{i-1} p+p_{i-1} q} \leq-1,
$$

which implies $\frac{p}{q} \in\left|\frac{p_{i-1}-p_{i-2}}{q_{i-1}-q_{i-2}}, \frac{p_{i-1}}{q_{i-1}}\right|$. It is easy to see that $\frac{p_{i-1}-p_{i-2}}{q_{i-1}-q_{i-2}} \in$ $\left|\frac{p_{i-3}}{q_{i-3}}, \frac{p_{i-1}}{q_{i-1}}\right|$. Hence by Lemma 1.1 we have that either $p_{i-1} / q_{i-1}$ or $p_{i-3} / q_{i-3}$ is a convergent of $p / q$. Thus, if we denote by $r$ the number of partial quotients (or convergents) of $p / q$, we have that $i-3 \leq r-1$. 
Now suppose $\gamma^{-1}(\infty)=\infty$. Then we have that, for all $i \geq 1$,

$$
\gamma_{i, x} \gamma^{-1}(y)=\gamma_{i, x}(x) \quad \text { and } \quad \gamma_{i, x} \gamma^{-1}(\infty)=\gamma_{i, x}(\infty) \text {, }
$$

SO

$$
\gamma_{i, x} \gamma^{-1}(y)>1 \quad \text { and } \quad-1 \leq \gamma_{i, x} \gamma^{-1}(\infty) \leq 0
$$

If, moreover, $\gamma_{i, x} \gamma^{-1}(\infty) \notin\{-1,0\}$, then, by Proposition 1.2, we have that $\gamma_{i, x} \gamma^{-1} \in \Gamma(y)$. Now, if $\gamma_{i, x}(\infty)=0$, then $\gamma_{i, x}=\gamma_{1, x}=\left(\begin{array}{cc}0 & 1 \\ 1 & -n_{0}\end{array}\right)$. If $\gamma_{i, x}(\infty)=-1$, then $n_{1}=1$ and $\gamma_{i, x}=\gamma_{2, x}=\left(\begin{array}{cc}1 & -n_{0} \\ -1 & 1+n_{0}\end{array}\right)$.

Therefore $i \leq 2$.

Remark 2.3. Let $\gamma \in \Gamma$ and set $\gamma^{-1}(\infty)=\left[n_{0}, \ldots, n_{r-1}\right]$. One can construct many real numbers $x$ for which $x_{r+1}$ is not a complete quotient of $\gamma(x)$ by letting $x=\left[n_{0}, \ldots, n_{r-2}, n_{r-1}-1,1, n_{r+1}, \ldots\right]$ where $n_{r+i}$ are arbitrary for all $i \geq 1$. Indeed, for such an $x$, we have that $\gamma^{-1}(\infty)=$ $p_{r} / q_{r}$, so $\gamma_{r+1, x} \gamma^{-1}(\infty)=\infty$, and then $\gamma_{r+1, x} \notin \Gamma(y) \gamma$. Therefore $x_{r+1}$ is not a complete quotient of $\gamma(x)$. Moreover, $\gamma_{r+2, x} \gamma^{-1}(\infty)=0$, and if $\operatorname{det}(\gamma)=\operatorname{det}\left(\gamma_{r+2, x}\right)$, then $\gamma_{r+2, x} \notin \Gamma(y) \gamma$ and $x_{r+2}$ is not a complete quotient of $\gamma(x)$ either.

\section{REFERENCES}

[KU10] Katok, S.; Ugarcovici, I.: Structure of attractors for $(a, b)$-continued fraction transformations, Journal of Modern Dynamics, 4 (2010), 637 - 691.

[Ser66] Serret, J.A.: Cours d'algèbre supérieure, 3ème ed., volume premier (1866).

Department of Mathematics, University of York, York, YO10 5DD, UNITED KINGDOM

E-mail address: paloma.bengoechea@york.ac.uk 